\title{
Role of CD14 in Lung Inflammation and Infection
}

\author{
A. Anas, T. van der Poll, and A.F. De Vos
}

\section{Introduction}

Toll-like receptors (TLR) on the surface of cells of the respiratory tract play an essential role in sensing the presence of microorganisms in the airways and lungs. These receptors trigger inflammatory responses, activate innate immune responses, and prime adaptive immune responses to eradicate invading microbes [1]. TLR are members of a family of pattern-recognition receptors, which recognize molecular structures of bacteria, viruses, fungi and protozoa (pathogen-associated molecular patterns or PAMPs), as well as endogenous structures and proteins released during inflammation (damage/danger-associated molecular patterns or DAMPs). To date, ten different TLR have been identified in humans and twelve in mice. TLR are expressed on all cells of the immune system, but also on parenchymal cells of many organs and tissues. The binding of a PAMP to a TLR results in cellular activation and initiates a variety of effector functions, including cytokine secretion, proliferation, co-stimulation or phagocyte maturation. To facilitate microbial recognition and to amplify cellular responses, certain TLR require additional proteins, such as lipopolysaccharide (LPS) binding protein (LBP), CD14, CD36 and high mobility group box-1 protein (HMGB-1). In this chapter, the role of CD14 as an accessory receptor for TLR in lung inflammation and infection is discussed. The central role of CD14 in the recognition of various PAMPs and amplification of immune and inflammatory responses in the lung is depicted in Figure 1.

CD14 was characterized as a receptor for bacterial endotoxin (LPS) in 1990, almost a decade before the discovery and characterization of TLR, and can be regarded as the first described pattern-recognition receptor [2]. The protein was first identified as a differentiation marker on the surface of monocytes and macrophages and was designated CD14 at the first leukocyte typing workshop in Paris in 1982. The genomic DNA of human CD14 was cloned in 1988 and the gene was later mapped to chromosome 5q23-31. Several polymorphisms have been found in the CD14 gene, of which nucleotide polymorphisms at position -159 and -1619 correlated with decreased lung function in endotoxin-exposed farmers [3].

The CD14 gene consists of two exons which code for a single mRNA that is translated into a protein of 375 amino acids. The CD14 protein is composed of eleven leucin-rich repeats, which are also found in TLR and which are important in PAMP binding. Moreover, the crystal structure of CD14 revealed that the protein has a 'horse-shoe' shape, similar to TLR4, and that LPS is bound within the pocket [4]. In contrast to TLR, however, CD14 lacks a transmembrane domain, and thus cannot initiate intracellular signal transduction by itself. The CD14 protein is processed in the endoplasmatic reticulum and expressed as a $55 \mathrm{kDa}$ glycoprotein on the cell sur- 


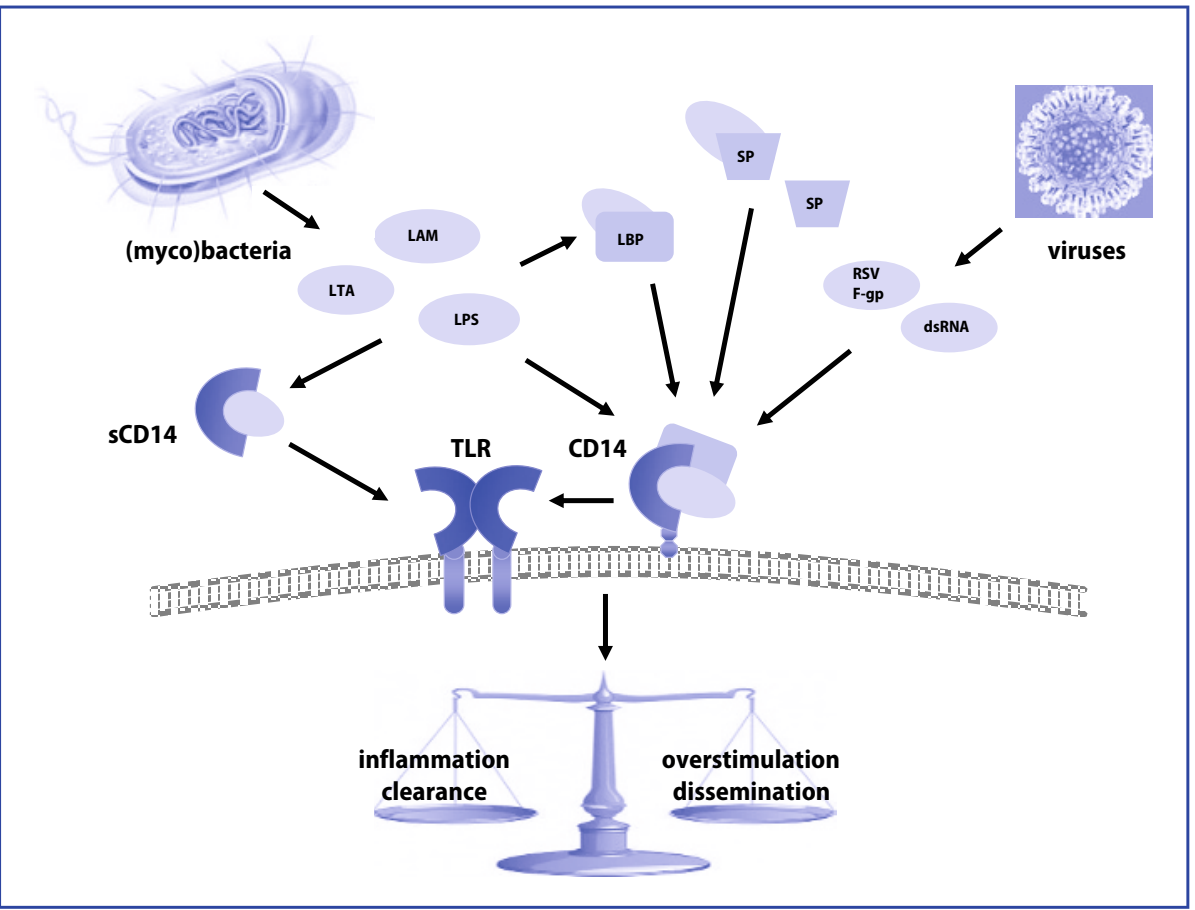

Fig. 1. Central role of CD14 in pathogen- and pathogen-associated molecular pattern (PAMP)-induced responses in the lung. CD14, which lacks an intracellular domain for signal transduction, is expressed on the surface of alveolar macrophages, infiltrating monocytes and neutrophils, and at lower levels also on epithelial and endothelial cells in the lung. CD14 recognizes and binds various structures from invading microbes, such as lipopolysaccharide (LPS) from Gram-negative bacteria, lipoteichoic acid (LTA) from Grampositive bacteria, lipoarabinomannan (LAM) from mycobacteria, viral double stranded (ds) RNA and F glycoprotein (F-gp) from respiratory syncytial virus (RSV). CD14 subsequently transfers these bound components to Toll-like receptors (TLR) which than trigger cell activation. Binding of LPS to CD14 is regulated by additional accessory receptors in the lung, including LPS-binding protein (LBP) and a number of surfactant proteins (SP). Furthermore, soluble CD14 (sCD14) enhances LPS-induced activation of cells with low CD14 expression. Depending on the microbe and the PAMPs it expresses, CD14-amplified responses can either be beneficial to the host by induction of an adequate inflammatory and immune response to eradicate the invading microbe, or detrimental to the host by excessive inflammation and/or dissemination of the pathogen.

face via a glycosylphosphatidyl (GPI) anchor [5]. Like other GPI-anchored proteins, CD14 accumulates on the cell surface in microdomains known as lipid rafts, which are fairly rich in cholesterol and accumulate several kinases at the intracellular site. CD14 is expressed predominantly on the surface of 'myeloid' cells, such as monocytes, macrophages and neutrophils, but at lower levels also on epithelial cells, endothelial cells and fibroblasts.

In addition to being expressed as a GPI-anchored membrane protein, CD14 is also expressed in a soluble form (sCD14) [2]. sCD14 may result from secretion of the protein before coupling to the GPI anchor or from shedding or cleavage from the surface of monocytes. sCD14 is present in the circulation and other body fluids and levels of sCD14 in plasma increase during inflammation and infection. Since inter- 
leukin (IL)-6 induces sCD14 expression in liver cells it is regarded as an acute phase protein. In bronchoalveolar lavage (BAL) fluid from patients with acute respiratory distress syndrome (ARDS), sCD14 levels were strongly increased and correlated with total protein levels and neutrophil numbers in the BAL fluid [6], suggesting that sCD14 contributes to the inflammatory process in the lung.

CD14 is a molecule with a wide range of functions. In addition to functioning as a pattern recognition receptor for a variety of microbial ligands, CD14 also acts as a receptor for endogenous molecules like intercellular adhesion molecule (ICAM)-3 on the surface of apoptotic cells, amyloid peptid, ceramide, and urate crystals. Ligation of CD14 by these ligands, except for apoptotic cells, mediates activation of inflammatory responses.

\section{CD14 and the LPS Receptor Complex}

LPS is the major constituent of the outer membrane of Gram-negative bacteria and is one of the most potent TLR ligands. CD14 together with LBP plays an essential role in binding of LPS to the TLR4/MD-2 complex [7]. LBP, which, among others, is present in the bloodstream and BAL fluid [8], binds to LPS aggregates and transfers LPS monomers to CD14. CD14 associates with TLR4/MD-2 and transfers the LPS monomer to this complex [7]. Likewise, sCD14 is able to mediate LPS-activation of cells with low membrane CD14 expression, such as epithelial and endothelial cells [9]. However, at high concentrations, LBP and sCD14 are also able to downregulate LPS-induced responses by transfer of LPS to lipoproteins for subsequent removal [10]. Recent data indicate that LPS is bound by MD-2 within the TLR4/MD-2 complex [11] and that subsequent conformational changes in TLR4 lead to reorganization of its cytoplasmic domain, enabling the recruitment of the adaptor proteins, myeloid differentiation primary-response protein 88 (MyD88) and TIR-domain-containing-adaptor-protein-inducing-interferon (IFN)- $\beta$ (TRIF) [12]. These adaptors initiate signal transduction to the nucleus by activation of nuclear factor (NF)- $\kappa B$ and IFN regulatory transcription factor (IRF)-3, leading to the production of cytokines that regulate inflammatory cells [12]. In macrophages, TRIF-dependent signaling is essential for the expression of the majority of LPS-induced genes, including IFN- $\alpha / \beta$.

Recently, it was reported that, in the absence of CD14, the TLR4/MD-2 complex can distinguish between different chemotypes of LPS [13]. Smooth LPS is synthesized by most Gram-negative bacteria and consists of three modules: The lipid A moiety, a core polysaccharide, and an O-polysaccharide of variable length (made up of 1 to over 50 monosaccharide units) [7]. Gram-negative bacteria that fail to add the core polysaccharide or the O-polysaccharide chain to the lipid A moiety produce 'rough' LPS, named after the rough morphology of the colonies these bacteria form. Lipid A, the bioactive part of both smooth and rough LPS, is responsible for most of the pathogenic effects in Gram-negative bacterial infections [7, 12]. Murine macrophages lacking CD14 secreted equal amounts of tumor necrosis factor- $\alpha$ (TNF) to macrophages expressing CD14 upon stimulation with rough LPS, but failed to secrete TNF in response to smooth LPS, an effect which was reversed by addition of sCD14 [13]. Moreover, macrophages lacking CD14 failed to secrete IFN- $\alpha / \beta$ in response to either rough or smooth LPS. These findings indicate that CD14 is required for activation of the TLR4/TRIF pathway by either smooth or rough LPS, and required for the activation of TLR4/MyD88 pathway by smooth but not by 
rough LPS [13]. In addition to LPS, CD14 also facilitates TLR4 activation by other PAMPs including certain viral components $[13,14]$.

In the lung, binding of LPS to TLR4 is influenced by a number of surfactant proteins (SP), including SP-A, SP-C and SP-D [15]. These surfactants are able to influence the interaction between TLR4 and LPS by direct binding to LPS; i.e., SP-A binds to rough LPS and lipid A, but not to smooth LPS, SP-C also binds to rough LPS, and SP-D binds to both rough and smooth LPS. SP-A and SP-C binding to LPS inhibits TNF secretion by alveolar macrophages, whereas SP-D binding to LPS moderately enhances TNF secretion by alveolar macrophages. In addition, SP-A, SP-C and SP-D also bind to CD14 at the site which recognizes LPS. Strikingly, binding of SP-A to CD14 enhanced the binding of rough LPS and binding of SP-C to CD14 augmented binding of smooth LPS [15], whereas binding of SP-A to CD14 reduced binding of smooth LPS and binding of SP-D to CD14 decreased binding of both smooth and rough LPS. Furthermore, SP-D influences LPS-induced TNF secretion by alveolar macrophages by regulating matrix metalloproteinase-mediated cleavage of CD14 from the surface of these cells [16].

Together, these findings suggest that LPS recognition in the lung and subsequent induction of inflammatory immune response is a complexly regulated process.

\section{CD14 and other Pattern Recognition Receptors}

In addition to LPS-induced activation of TLR4, CD14 also amplifies a number of TLR-dependent responses triggered by other bacterial PAMPs, including peptidoglycan, lipoteichoic acid (LTA) and lipoarabinomannan (LAM) [17-19].

Peptidoglycan is an essential cell wall component of virtually all bacteria. Peptidoglycan is a polymer of $\mathrm{N}$-acetylglucosamine and $\mathrm{N}$-acetylmuramic acid, crosslinked by short peptides. Breakdown products of peptidoglycan are recognized by different classes of pattern-recognition receptors [19]. Polymeric soluble peptidoglycan is recognized by TLR2 on the surface of cells, and the interaction of peptidoglycan with TLR2 triggers MyD88-dependent activation and nuclear translocation of $\mathrm{NF}-\kappa \mathrm{B}$, and subsequently the transcription and secretion of cytokines. Muramyl dipeptide and $\gamma$-D-glutamyl-meso-diaminopimelic acid, which are low-molecular weight breakdown fragments of peptidoglycan, are recognized by intracellular pathogen recognition receptors, nucleotide-binding oligomerization domain containing (Nod)2 and Nod1, respectively [19]. Ligand binding to these receptors triggers interaction with the receptor-interacting protein kinase, RIP2, which activates NF- $\kappa B$. Of these peptidoglycan breakdown products, only polymeric peptidoglycan binds to CD14, and CD14 enhances polymeric peptidoglycan-induced TLR2 activation. The low molecular weight fragments of peptidoglycan, like muramyl dipeptide, do not bind to CD14, do not induce cell activation through CD14 and also do not interfere with the binding of polymeric peptidoglycan to CD14 [19]. Furthermore, unlike LPS, peptidoglycan bound to sCD14 is not able to activate epithelial and endothelial cells with low membrane CD14 expression.

LTA is a constituent of the cell wall of Gram-positive bacteria, anchored on the outer face of the cytoplasmic membrane and commonly released during growth and antibiotic therapy. Like polymeric peptidoglycan, LTA induces NF- $\mathrm{KB}$ activation and cytokine secretion in a TLR2-dependent manner. LTA is recognized by LBP and CD14, and these accessory receptors both enhance LTA-induced cell activation [18]. Presumably in a similar manner, CD14 also enhances TLR2-dependent cellular acti- 
vation by LAM derived from the cell-wall of mycobacteria. LAM derived from slowly growing virulent mycobacteria like Mycobacterium tuberculosis and M. leprae is capped with mannose (ManLAM), whereas LAM from avirulent and fast growing mycobacterial species is uncapped (AraLAM). Strikingly, AraLAM from avirulent mycobacteria is much more potent in inducing TNF secretion by macrophages than ManLAM from virulent mycobacterial strains [12]. AraLAM-, but not ManLAMinduced TNF secretion by monocytes and macrophages was largely CD14-, TLR2and MyD88-dependent [17].

Recently CD14 was also found to enhance the innate immune response triggered by the TLR3 ligand poly(I:C), a synthetic mimic of double stranded RNA [20]. TLR3 together with TLR7 and TLR8 are regarded as sensors for viral infection, since these receptors recognize viral nucleic acids, like single and double stranded RNA. The potentiating effect of CD14 on TLR3 activation resulted from increased uptake of poly(I:C) and intracellular delivery to the compartment where TLR3 resides [20]. Taken together, these findings suggest that CD14 plays an important role in the induction and amplification of inflammatory responses evoked by a wide variety of pathogens.

\section{Role of CD14 in LPS- and LTA-induced Lung Inflammation}

The contribution of CD14 to TLR ligand-induced lung inflammation has been investigated in several animal studies (Table 1). Intratracheal administration of LPS did not significantly induce TNF release and neutrophil accumulation in the lungs of rabbits, unless LPS was complexed with LBP [21] or the animals were subjected to mechanical ventilation [22]. Intratracheal instillation of anti-CD14 antibodies together with LPS/LBP or intravenous pretreatment with anti-CD14 or anti-TLR4 antibodies before mechanical ventilation markedly reduced these inflammatory responses $[21,22]$. Despite a reduction in lung neutrophil number, intravenous antiCD14 treatment of rabbits exposed to LPS and subjected to ventilation did not cause a decrease in lung chemokines, including CXCL8 (IL-8), growth related oncogene (GRO) and monocyte chemoattractant protein (MCP)-1, whereas anti-TLR4 treatment did lower the level of GRO moderately and of CXCL8 significantly [22]. These findings reveal that LPS alone does not cause significant lung inflammation in rabbits and suggest that additional accessory signals are required. Whether mechanical ventilation induces increased release of LBP or release of (endogenous) DAMPs which potentiate the LPS-induced response remains to be determined.

In contrast to rabbits, administration of LPS alone to lungs of naive mice induced severe pneumonitis, irrespective of the manner of LPS delivery (inhalation or intratracheal or intranasal instillation) or the source of LPS (Escherichia coli or Acinetobacter baumannii). Using antibody-treated and gene-deficient mice, CD14 was found to be critically involved in the development of LPS-induced lung inflammation [23-26]. A study with CD14-deficient mice and TLR4 mutant mice (lacking a functional TLR4) showed that LPS-induced vascular leakage, neutrophil infiltration, nuclear translocation of NF- $\mathrm{BB}$. The release of cytokines (TNF and IL-6) and chemokines (CXCL1 and CXCL2) in the lung was completely dependent on these pattern recognition receptors [24]. Similar observations were made by others using mice treated intravenously with anti-CD14 antibodies [23] and by our group using CD14deficient and TLR4-deficient mice [25]. Furthermore, intratracheal treatment of CD14-deficient mice with sCD14 restored the inflammatory response to the level 
Table 1. Effect of CD14 'neutralization' in lung inflammation and lung infection

\begin{tabular}{|c|c|c|c|}
\hline Inciting ligand/pathogen & Animal model* & Effect of CD14 'neutralization' in the lung** & Ref. \\
\hline LPS (E. coli +LBP) & rabbit $\alpha C D 14$ & $\downarrow \downarrow$ neutrophil influx, $\downarrow \downarrow$ cytokines & 21 \\
\hline LPS (E. coli +ventilation) & & $\downarrow \downarrow$ neutrophil influx, $\sim$ chemokines & 22 \\
\hline LPS (E. coli) & mouse $\alpha C D 14$ & $\begin{array}{l}\downarrow \text { neutrophil influx, } \downarrow \text { vascular leakage, } \\
\downarrow N F-\kappa B \text { activation }\end{array}$ & 23 \\
\hline LPS (E. coli) & mouse CD14\% & $\begin{array}{l}\downarrow \downarrow \text { neutrophil influx (reversed by } s C D 14 \text { ), } \\
\downarrow \downarrow \text { cytokines (restored by sCD14), } \downarrow \downarrow \text { chemokines, } \\
\downarrow \downarrow \text { vascular leakage }\end{array}$ & 24,26 \\
\hline LPS (A. baumannii) & & $\downarrow \downarrow$ neutrophil influx, $\downarrow \downarrow$ cytokines & 25 \\
\hline $\begin{array}{l}\text { LTA (S. aureus) } \\
\text { LTA (S. pneumoniae) }\end{array}$ & mouse $\mathrm{CD} 14^{-/-}$ & $\begin{array}{l}\sim \text { neutrophil influx, } \downarrow \text { cytokines, } \downarrow \text { chemokines } \\
\downarrow \text { neutrophil influx, } \sim \text { cytokines, } \sim \text { chemokines }\end{array}$ & $\begin{array}{l}28 \\
29\end{array}$ \\
\hline nontypeable $H$. influenza & mouse $\mathrm{CD} 14^{-/-}$ & $\begin{array}{l}\downarrow \downarrow \text { clearance, } \downarrow \downarrow \text { (early) } \uparrow \uparrow(\text { late) neutrophil influx, } \\
\downarrow \downarrow \text { (early) } \uparrow \uparrow \text { (late) cytokines }\end{array}$ & 30 \\
\hline A. baumannii & mouse CD14 $4^{--}$ & $\begin{array}{l}\downarrow \downarrow \text { clearance, } \sim \text { neutrophil influx, } \\
\sim \text { cytokines ( } \uparrow \text { dissemination) }\end{array}$ & 25 \\
\hline E. coli & rabbit $\alpha C D 14$ & $\begin{array}{l}\downarrow \downarrow \text { clearance, } \sim \text { neutrophil influx, } \sim \text { cytokines, } \\
\sim \text { chemokines }(\downarrow \downarrow \text { systemic responses })\end{array}$ & 32 \\
\hline B. pseudomallei & mouse $\mathrm{CD} 14^{--}$ & $\begin{array}{l}\uparrow \uparrow c l e a r a n c e \text { (reversed by sCD14), } \downarrow \text { neutrophil influx } \\
\text { (reversed by sCD14), } \sim \text { cytokines ( } \uparrow \text { systemic clear- } \\
\text { ance (reversed by sCD14)) ( } \downarrow \downarrow \text { mortality) }\end{array}$ & 40 \\
\hline S. pneumoniae & mouse $\mathrm{CD} 14^{-/-}$ & $\begin{array}{l}\uparrow \uparrow c l e a r a n c e \text { (reversed by sCD14), } \downarrow \downarrow \text { neutrophil } \\
\text { influx, } \downarrow \downarrow \text { cytokines, } \downarrow \downarrow \text { chemokines } \\
\text { ( } \downarrow \downarrow \text { dissemination (reversed by sCD14)) ( } \downarrow \downarrow \text { mortal- } \\
\text { ity (reversed by sCD14)) }\end{array}$ & 41 \\
\hline M. tuberculosis & mouse $\mathrm{CD} 14^{--}$ & $\begin{array}{l}\sim \text { clearance, } \downarrow \downarrow \text { cellular infiltration, } \sim / \uparrow \text { cytokines } \\
(\downarrow \downarrow \text { mortality) }\end{array}$ & 44 \\
\hline Influenza A & mouse $\mathrm{CD} 14^{-/}$ & $\begin{array}{l}\uparrow / \sim \text { clearance, } \sim \text { lymphocyte recruitment and } \\
\text { activation, } \sim \text { neutrophil influx, } \sim \text { cytokines }\end{array}$ & 50 \\
\hline
\end{tabular}

* $\alpha$ CD14: anti-CD14 antibody treatment; CD14 ${ }^{--}$: CD14-gene deficient

** $\downarrow(\downarrow)$ : (strongly) reduced; $\sim$ : unaltered; $\uparrow(\uparrow)$ : (strongly) increased

LPS: lipopolysaccharide; LTA: lipoteichoic acid

present in wild-type mice, whereas treatment with wild-type alveolar macrophages restored the neutrophil infiltration of the lung but not pulmonary TNF release [26]. Moreover, treatment with wild-type alveolar macrophages also restored neutrophil infiltration in the lung of LPS-exposed TLR4-deficient mice [27]. These findings indicate that sCD14, and CD14 and TLR4 on the surface of alveolar macrophages contribute to the development of LPS-induced lung inflammation. However, when a high dose of LPS was administered to the lungs of mice, acute lung inflammation was absent in mice lacking functional TLR4, but only partially reduced in CD14 deficient mice [24]. Thus, LPS-induced lung inflammation is entirely dependent on TLR4 and, depending on the dose of LPS, also on the presence of CD14 in the lung.

Our group determined whether CD14 also contributes to the development of lung inflammation induced by LTA, a TLR2 ligand from the cell wall of Gram-positive bacteria [28, 29]. Lung inflammation induced by Staphylococcus aureus LTA was completely dependent on TLR2, but independent of LBP and only moderately 
dependent on CD14 expression. As compared to wild-type mice, S. aureus LTAinduced neutrophil influx was unchanged in CD14-deficient mice, whereas TNF and CXCL2 release in the lung were partially reduced [28]. Strikingly, however, pulmonary inflammation was also greatly diminished in TLR4-deficient mice, as well as in mice deficient for platelet activating factor receptor (PAFR), a known receptor for LTA on epithelial cells. Similarly, lung inflammation induced by Streptococcus pneumoniae LTA, which is less potent compared S. aureus LTA, was also completely dependent on TLR2 expression. However, in contrast to $S$. aureus LTA, neutrophil infiltration of the lung was moderately reduced in CD14-deficent mice treated with pneumococcal LTA, whereas TNF and CXCL2 release in the lung was unchanged [29]. Moreover, pneumococcal LTA-induced lung inflammation was moderately diminished in TLR4-deficient mice. Thus, despite the amplifying effect on LTAinduced TLR2-mediated responses in vitro, CD14 contributes minimally to lung inflammation induced by LTA. The unexpected contribution of TLR4 to LTA-induced lung inflammation may result from DAMPs generated during the inflammatory process in the respiratory tract.

\section{Role of CD14 in Lung Infection}

In line with the findings that CD14 contributes to LPS-induced lung inflammation in mice, a number of studies have shown that CD14 is essential for the host defense response in the lung against Gram-negative bacteria, such as nontypeable Haemophilus influenzae, a possible cause of community acquired pneumonia, and A. baumannii and $E$. coli, which are frequent inducers of nosocomial pneumonia (Table 1). Nontypeable $H$. influenzae expresses the TLR4 ligands LPS and lipooligosaccharide on its cell wall, as well as several TLR2 ligands, including lipoproteins and porins. Previously, we found that activation of alveolar macrophages by nontypeable $H$. influenzae depended on expression of TLR4, TLR2, and CD14 [30]. Moreover, bacterial clearance after intranasal infection with nontypeable $H$. influenzae was markedly reduced in CD14-deficient and TLR4-deficient mice, as well as in TLR2-deficient mice at later stages of the disease [30]. Interestingly, despite impaired bacterial clearance in CD14-deficient and TLR4-deficient mice, the inflammatory response in the lung was strongly reduced in TLR4 deficient mice, but elevated in CD14 deficient mice. Similar observations were made with encapsulated $H$. influenzae in TLR4mutant mice [31]. Furthermore, clearance of nontypeable $H$. influenzae was also significantly impaired in MyD88-deficient mice, but not in mice lacking functional TRIF [30]. In a similar manner, CD14 was involved in the host defense response against $A$. baumanii [25]. CD14-deficient mice, like-TLR4-deficient mice, suffered from impaired bacterial clearance in the lungs and enhanced bacterial dissemination after intranasal infection with A. baumannii. However, unlike TLR4-deficient mice, CD14-deficient mice developed similar inflammatory responses compared to wild-type mice. These findings suggest a role for CD14 in anti-bacterial responses against nontypeable $H$. influenzae and A. baumannii. Although the role of TLR4 (and TLR2) in phagocytic killing is controversial, it is unknown whether CD14 is involved in such processes. The role of CD14 in E. coli-induced pneumonia was determined in anti-CD14 antibody treated rabbits. Intravenous anti-CD14 antibody treatment of rabbits inoculated with $E$. coli by bronchial instillation, resulted in decreased bacterial clearance from the lungs, but had no effect on neutrophil infiltration or cytokine release in the lungs [32]. However, anti-CD14 treatment pro- 
tected against sustained hypotension and reduced the levels of nitrate and nitrite in the blood. The contribution of CD14 to E. coli-induced pneumonia has not been investigated in mice, whereas the role of the other components of the LPS receptor complex (TLR4, MD-2, MyD88, TRIF) has been determined using gene-deficient or mutant mice. Although analysis of bacterial clearance after intranasal infection of TLR4-mutant mice with E. coli produced inconsistent results [33], lack of MD-2 or TRIF resulted in impaired bacterial clearance after $E$. coli instillation in the lungs [34, 35]. Moreover, E. coli-induced neutrophil accumulation and cytokine release was significantly reduced in mice devoid of functional TLR4, MD-2, MyD88 or TRIF [33-35]. These findings indicate that signaling through the TLR4 receptor complex is essential in the host defense response against E. coli, and suggests that CD14 may contribute to these $E$. coli-induced responses.

To our knowledge, it is unclear whether CD14 contributes to host defense against Pseudomonas aeruginosa, a frequent cause of nosocomial pneumonia, and Burkholderia cepacia, a prevalent Gram-negative bacterium, together with P. aeruginosa, in patients with cystic fibrosis. Recently, it was found that both TLR4 and TLR5 are critical in the host response to P. aeruginosa and that TLR4-deficient mice were not susceptible to intratracheal $P$. aeruginosa infection unless a bacterial mutant devoid of flagellin production was used [36]. A similar approach is required to determine a role for CD14 in Pseudomonas-induced pneumonia. It is plausible that CD14 also contributes to the host response against $B$. cepacia, since LPS from this bacterium signals through TLR4 and anti-CD14 antibodies dramatically inhibited B. cepaciainduced chemokine secretion by lung epithelial cells [37]. Whether CD14 contributes to host defense response against Klebsiella pneumoniae, a known cause of nosocomial pneumonia, also remains to be determined, but data from our study with TLR4-mutant mice indicate that signaling through TLR4 is essential for successful clearance of this bacterium [38].

In contrast to the essential role of pulmonary TLR4 and CD14 in the host defense response against most Gram-negative bacteria, we found that TLR4 was not involved and CD14 played a remarkable detrimental role in the host response to B. pseudomallei, the causative organism of melioidosis (the most common cause of community-acquired sepsis in Southeast Asia) [39, 40]. CD14-deficient mice infected intranasally with $B$. pseudomallei were protected from mortality, accompanied by enhanced bacterial clearance in the lung, blood and liver, and reduced cellular infiltration in the lung [39], whereas the course of disease in TLR4-deficient mice was indistinguishable from wild-type mice [40]. Moreover, intranasal administration of sCD14 to CD14-deficient mice partially reversed the phenotype into that of wildtype mice [40]. Interestingly, these findings in B. pseudomallei-infected CD14-deficient mice strongly resemble our previous results found with TLR2-deficient mice, and are in line with the observation that $B$. pseudomallei expresses an atypical LPS which signals through TLR2 [39]. Whether CD14 interacts with TLR2 in B. pseudomallei-induced responses, and by which mechanism these receptors facilitate the growth and dissemination of $B$. pseudomallei after intranasal infection remains to be determined.

In the model for S. pneumoniae-induced pneumonia, we observed an unexpected detrimental role for CD14 in the innate host defense response. S. pneumoniae, a Gram-positive bacterium and the single most frequent pathogen causing community-acquired pneumonia, induces severe lung inflammation and sepsis in wild-type mice after intranasal instillation. Strikingly, CD14-deficient mice were protected against pneumococcal pneumonia, presumably as a result of reduced bacterial 
spread to the circulation and reduced lung inflammation [41]. In contrast, TLR2deficient and TLR4-mutant mice were not protected against pneumococcal pneumonia [38, 42], but in fact TLR2 seemed redundant for efficient bacterial clearance and TLR4-mutant mice were more susceptible to pneumonia, accompanied by impaired bacterial clearance. However, as in CD14-deficient mice, lung inflammation was also reduced in pneumococci-infected TLR2-deficient mice [42]. Since intrapulmonary treatment with sCD14 rendered CD14-deficient mice equally susceptible to S. pneumoniae as wild-type mice [41], these results suggest that $S$. pneumoniae abuses (s)CD14 in the lung to cause invasive respiratory tract infection. Interestingly, the phenotype of CD14 deficient mice strongly resembled the phenotype of mice deficient for PAFR [43], a receptor for phosphoryl choline from the pneumococcal cell wall which facilitates pneumococcal invasion of cells. Further studies are required to determine whether CD14 serves as a chaperone in the presentation of $S$. pneumoniae to the PAFR so that the phosphoryl-PAFR-mediated invasion is facilitated.

Since $M$. tuberculosis expresses a number of molecules, such as lipoproteins, which activate immune cells in a CD14-dependent manner, we and others investigated whether CD14 also contributed to the host immune response in mice with lung tuberculosis [44]. Although initially after intranasal infection of wild-type and CD14-deficient mice no differences in bacterial loads, cell infiltration and release of most cytokines in the lung were found $[44,45]$, at later time points $(>20$ weeks after infection) CD14-deficient mice were protected from mortality presumably as a result of a reduced inflammatory response in the lungs [44]. These findings are completely opposite to the results from $M$. tuberculosis-infected TLR2-deficient and TLR4mutant mice, which suffered from reduced bacterial clearance, chronic inflammation, increased cellular infiltration of the lungs and reduced survival [46-48]. The mechanism underlying the detrimental effect of CD14 in the host response against M. tuberculosis remains to be established.

In addition to its role in (myco)bacterial infections, CD14 may also play a role in the pulmonary host response against respiratory syncytial virus (RSV), the most common cause of lower respiratory tract disease in infants and young children worldwide, and influenza A virus, a cause of pneumonia in very young children, the elderly and immunocompromised patients. The envelop F glycoprotein from RSV and certain influenza A virus components activate macrophages in a CD14-dependent manner $[14,20]$. Experiments with wild-type and TLR4-mutant mice infected intranasally with RSV showed that viral clearance was reduced in the absence of functional TLR4 [14], due to impaired natural killer (NK) cell migration and function and impaired cytokine secretion. Recently, it was found that TLR2 and TLR6 are also involved in recognition of RSV [49]. Whether CD14 contributes to these TLR-mediated immune responses against RSV remains to be determined. Using CD14-deficient mice, we demonstrated that CD14 played a minimal role in influenza A virus-induced pneumonia [50]. During the entire course of disease, viral loads were slightly reduced in CD14-deficient mice, but this did not result from improved lymphocyte recruitment or lymphocyte activation, or consistent changes in pulmonary cytokines [50]. Thus, despite the fact that influenza A expresses ligands that require CD14 for immune cell activation [20], CD14 seems redundant in the host defense reponse against influenza A virus. 


\section{Conclusion}

CD14 plays a central role in the lung in the recognition and binding of a variety of (myco)bacterial and viral components, and in the amplification of subsequent host responses. The studies discussed in this chapter indicate that the contribution of CD14 to the pulmonary host defense responses may range from beneficial to detrimental, depending on the microbe and the PAMPs it expresses. Interfering with CD14-LPS or CD14-LTA interactions reduced lung inflammation. Interference with CD14-pathogen interactions, however, did not have a significant effect on $M$. tuberculosis or influenza A virus infection, resulted in reduced clearance of nontypeable $H$. influenzae, E. coli or A. baumannii in the lung, but enhanced clearance (and reduced dissemination) of $B$. pseudomallei or S. pneumoniae. The latter observation indicates that certain pathogens may abuse CD14 in the lung to cause invasive disease. Whether CD14 is a suitable target for intervention in these latter infectious diseases and/or in aberrant inflammatory responses during pneumonia requires further study.

\section{References}

1. Basu S, Fenton MJ (2004) Toll-like receptors: function and roles in lung disease. Am J Physiol Lung Cell Mol Physiol 286:L887-L892

2. Wright SD (1995) CD14 and innate recognition of bacteria. J Immunol 155: 6-8

3. LeVan TD, Von Essen S, Romberger DJ, et al (2005) Polymorphisms in the CD14 gene associated with pulmonary function in farmers. Am J Respir Crit Care Med 171: 773-779

4. Kim JI, Lee CJ, Jin MS, et al (2005) Crystal structure of CD14 and its implications for lipopolysaccharide signaling. J Biol Chem 280: 11347-11351

5. Wright SD, Ramos RA, Tobias PS, Ulevitch RJ, Mathison JC (1990) CD14, a receptor for complexes of lipopolysaccharide (LPS) and LPS binding protein. Science 249: 1431-1433

6. Martin TR, Rubenfeld GD, Ruzinski JT, et al (1997) Relationship between soluble CD14, lipopolysaccharide binding protein, and the alveolar inflammatory response in patients with acute respiratory distress syndrome. Am J Respir Crit Care Med 155: 937-944

7. Beutler B, Rietschel ET (2003) Innate immune sensing and its roots: the story of endotoxin. Nat Rev Immunol 3: 169-176

8. Knapp S, Florquin S, Golenbock DT, Van der Poll T (2006) Pulmonary lipopolysaccharide (LPS)-binding protein inhibits the LPS-induced lung inflammation in vivo. J Immunol 176: 3189-3195

9. Pugin J, Schurer-Maly CC, Leturcq D, et al (1993) Lipopolysaccharide activation of human endothelial and epithelial cells is mediated by lipopolysaccharide-binding protein and soluble CD14. Proc Natl Acad Sci USA 90: 2744-2748

10. Kitchens RL, Thompson PA (2005) Modulatory effects of sCD14 and LBP on LPS-host cell interactions. J Endotoxin Res 11: 225-229

11. Park BS, Song DH, Kim HM, et al (2009) The structural basis of lipopolysaccharide recognition by the TLR4-MD-2 complex. Nature 458: 1191-1195

12. Akira S, Uematsu S, Takeuchi O (2006) Pathogen recognition and innate immunity. Cell 124: $783-801$

13. Jiang Z, Georgel P, Du X, et al (2005) CD14 is required for MyD88-independent LPS signaling. Nat Immunol 6: 565-570

14. Kurt-Jones EA, Popova L, Kwinn L, et al (2000) Pattern recognition receptors TLR4 and CD14 mediate response to respiratory syncytial virus. Nat Immunol 1: 398-401

15. Chaby R, Garcia-Verdugo I, Espinassous Q, Augusto LA (2005) Interactions between LPS and lung surfactant proteins. J Endotoxin Res 11: 181-185

16. Senft AP, Korfhagen TR, Whitsett JA, Shapiro SD, LeVine AM (2005) Surfactant protein-D regulates soluble CD14 through matrix metalloproteinase-12. J Immunol 174: 4953-4959

17. Pugin J, Heumann ID, Tomasz A, et al (1994) CD14 is a pattern recognition receptor. Immu nity 1: $509-516$ 
18. Schroder NW, Morath S, Alexander C, et al (2003) Lipoteichoic acid (LTA) of Streptococcus pneumoniae and Staphylococcus aureus activates immune cells via Toll-like receptor (TLR)2, lipopolysaccharide-binding protein (LBP), and CD14, whereas TLR-4 and MD-2 are not involved. J Biol Chem 278: 15587-15594

19. Dziarski R, Gupta D (2005) Peptidoglycan recognition in innate immunity. J Endotoxin Res 11: $304-310$

20. Lee HK, Dunzendorfer S, Soldau K, Tobias PS (2006) Double-stranded RNA-mediated TLR3 activation is enhanced by CD14. Immunity 24: 153-163

21. Ishii Y, Wang Y, Haziot A, et al (1993) Lipopolysaccharide binding protein and CD14 interaction induces tumor necrosis factor-alpha generation and neutrophil sequestration in lungs after intratracheal endotoxin. Circ Res 73: 15-23

22. Smith LS, Kajikawa O, Elson G, et al (2008) Effect of Toll-like receptor 4 blockade on pulmonary inflammation caused by mechanical ventilation and bacterial endotoxin. Exp Lung Res 34: $225-243$

23. Tasaka S, Ishizaka A, Yamada W, et al (2003) Effect of CD14 blockade on endotoxin-induced acute lung injury in mice. Am J Respir Cell Mol Biol 29: 252-258

24. Jeyaseelan S, Chu HW, Young SK, Freeman MW, Worthen GS (2005) Distinct roles of pattern recognition receptors CD14 and Toll-like receptor 4 in acute lung injury. Infect Immun 73: $1754-1763$

25. Knapp S, Wieland CW, Florquin S, et al (2006) Differential roles of CD14 and toll-like receptors 4 and 2 in murine Acinetobacter pneumonia. Am J Respir Crit Care Med 173: 122-129

26. Brass DM, Hollingsworth JW, McElvania-Tekippe E, et al (2007) CD14 is an essential mediator of LPS induced airway disease. Am J Physiol Lung Cell Mol Physiol 293: L77-83

27. Hollingsworth JW 2nd, Cook DN, Brass DM, et al (2004) The role of Toll-like receptor 4 in environmental airway injury in mice. Am J Respir Crit Care Med 170: 126-132

28. Knapp S, von Aulock S, Leendertse M, et al (2008) Lipoteichoic acid-induced lung inflammation depends on TLR2 and the concerted action of TLR4 and the platelet-activating factor receptor. J Immunol 180: 3478-3484

29. Dessing MC, Schouten M, Draing C, et al (2008) Role played by Toll-like receptors 2 and 4 in lipoteichoic acid-induced lung inflammation and coagulation. J Infect Dis 197: 245-252

30. Wieland CW, Florquin S, Maris NA, et al (2005) The MyD88-dependent, but not the MyD88independent, pathway of TLR4 signaling is important in clearing nontypeable haemophilus influenzae from the mouse lung. J Immunol 175: 6042-6049

31. Wang X, Moser C, Louboutin JP, et al (2002) Toll-like receptor 4 mediates innate immune responses to Haemophilus influenzae infection in mouse lung. J Immunol 168: 810-815

32. Frevert CW, Matute-Bello G, Skerrett SJ, et al (2000) Effect of CD14 blockade in rabbits with Escherichia coli pneumonia and sepsis. J Immunol 164: 5439-5445

33. Lee JS, Frevert CW, Matute-Bello G, et al (2005) TLR-4 pathway mediates the inflammatory response but not bacterial elimination in E. coli pneumonia. Am J Physiol Lung Cell Mol Physiol 289:L731-L738

34. Jeyaseelan S, Young SK, Fessler MB, et al (2007) Toll/IL-1 receptor domain-containing adaptor inducing IFN-beta (TRIF)-mediated signaling contributes to innate immune responses in the lung during Escherichia coli pneumonia. J Immunol 178: $3153-3160$

35. Cai S, Zemans RL, Young SK, Worthen GS, Jeyaseelan S (2009) Myeloid differentiation protein-2-dependent and -independent neutrophil accumulation during Escherichia coli pneumonia. Am J Respir Cell Mol Biol 40: 701 - 709

36. Ramphal R, Balloy V, Jyot J, et al (2008) Control of Pseudomonas aeruginosa in the lung requires the recognition of either lipopolysaccharide or flagellin. J Immunol 181: 586-592

37. Reddi K, Phagoo SB, Anderson KD, Warburton D (2003) Burkholderia cepacia-induced IL-8 gene expression in an alveolar epithelial cell line: signaling through CD14 and mitogen-activated protein kinase. Pediatr Res 54: 297-305

38. Branger J, Knapp S, Weijer S, et al (2004) Role of Toll-like receptor 4 in gram-positive and gram-negative pneumonia in mice. Infect Immun 72: 788-794

39. Wiersinga WJ, Wieland CW, Dessing MC, et al (2007) Toll-like receptor 2 impairs host defense in gram-negative sepsis caused by Burkholderia pseudomallei (Melioidosis). PLoS Med 4: e248

40. Wiersinga WJ, de Vos AF, Wieland CW, et al (2008) CD14 impairs host defense against 
gram-negative sepsis caused by Burkholderia pseudomallei in mice. J Infect Dis 198: 13881397

41. Dessing MC, Knapp S, Florquin S, De Vos AF, Van der Poll T (2007) CD14 facilitates invasive respiratory tract infection by Streptococcus pneumoniae. Am J Respir Crit Care Med 175: 604-611

42. Knapp S, Wieland CW, Murawskian 't Veer C, et al (2004) Toll-like receptor 2 plays a role in the early inflammatory response to murine pneumococcal pneumonia but does not contribute to antibacterial defense. J Immunol 172: $3132-3138$

43. Rijneveld AW, Weijer S, Florquin S, et al (2004) Improved host defense against pneumococcal pneumonia in platelet-activating factor receptor-deficient mice. J Infect Dis 189: 711-716

44. Wieland CW, Van der Windt GJ, Wiersinga WJ, Florquin S, Van der Poll T (2008) CD14 contributes to pulmonary inflammation and mortality during murine tuberculosis. Immunology 125: $272-279$

45. Reiling N, Holscher C, Fehrenbach A, et al (2002) Toll-like receptor (TLR)2- and TLR4-mediated pathogen recognition in resistance to airborne infection with Mycobacterium tuberculosis. J Immunol 169: $3480-3484$

46. Abel B, Thieblemont N, Quesniaux VJ, et al (2002) Toll-like receptor 4 expression is required to control chronic Mycobacterium tuberculosis infection in mice. J Immunol 169: 3155-3162

47. Drennan MB, Nicolle D, Quesniaux VJ, et al (2004) Toll-like receptor 2-deficient mice succumb to Mycobacterium tuberculosis infection. Am J Pathol 164: 49-57

48. Branger J, Leemans JC, Florquin S, et al (2004) Toll-like receptor 4 plays a protective role in pulmonary tuberculosis in mice. Int Immunol 16: 509-516

49. Murawski MR, Bowen GN, Cerny AM, et al (2009) Respiratory syncytial virus activates innate immunity through Toll-like receptor 2. J Virol 83: 1492-1500

50. Dessing MC, Van der Sluijs KF, Florquin S, Van der Poll T (2007) CD14 plays a limited role during influenza A virus infection in vivo. Immunol Lett 113: 47-51 